\title{
Comment empêcher le SRAS-CoV-2 d'entrer dans les écoles
}

\author{
Nisha Thampi MD MSc, Beate Sander PhD, Michelle Science MD MSc
}

Citation : CMAJ 2020 January 4;193:E24-5. doi : 10.1503/cmaj.202568-f; diffusion hâtive le 24 novembre 2020

Voir la version anglaise de l'article ici : www.cmaj.ca/lookup/doi/10.1503/cmaj.202568; voir la recherche connexe en anglais ici : www. cmaj.ca/lookup/doi/10.1503/cmaj.202065 et en français ici : www.cmaj.ca/lookup/doi/10.1503/cmaj.202065-f

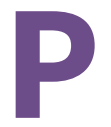

artout dans le monde, des mesures de santé et de sécurité renforcées ont été mises en place dans les écoles et lors d'activités de groupe pour prévenir la transmission du coronavirus du syndrome respiratoire aigu sévère 2 (SRAS-CoV-2) chez les élèves et le personnel. L'une de ces mesures, le dépistage des symptômes, vise à empêcher les personnes symptomatiques d'entrer dans les écoles. II se pose cependant une difficulté particulière du fait que les manifestations cliniques du SRAS-CoV-2 varient grandement d'un enfant à l'autre.

La connaissance des symptômes souvent associés à l'infection par le SRAS-CoV-2 pourrait faciliter l'établissement de critères de dépistage et de test pour les enfants. Une étude connexe fournit des renseignements utiles à cet égard ${ }^{1}$ : King et ses collègues ont cherché les symptômes les plus susceptibles d'annoncer l'infection par ce virus dans une cohorte d'enfants et d'adolescents (moins de 19 ans) de l'Alberta. Ils ont constaté qu'après un premier test effectué entre avril et septembre 2020, parmi les enfants ayant été déclarés positifs, $35,9 \%$ n'avaient déclaré aucun symptôme, soit une proportion supérieure aux estimations regroupées d'examens systématiques sur la maladie à coronavirus 2019 (COVID-19) dans la population pédiatrique, qui varient entre $15 \%$ et $20 \%^{2}$. Cet écart pourrait être dû à la vaste stratégie de test des contacts étroits adoptée en Alberta depuis le début d'avril, qui comprend le test des contacts asymptomatiques ou présymptomatiques à des moments précis après l'exposition.

L'étude connexe confirme également l'ubiquité des symptômes pouvant se rapporter à la COVID-19 chez les enfants et les adolescents. La fièvre, la toux et la rhinorrhée étaient les symptômes les plus courants chez les enfants ayant subi un test de dépistage de l'infection par le SRAS-CoV-2, indépendamment du résultat. En revanche, les symptômes les plus susceptibles d'être associés à un résultat positif étaient l'anosmie, l'agueusie, la nausée, les vomissements, le mal de tête, la fièvre et les frissons. Un récent examen général a recensé plus de 30 examens systématiques sur les manifestations cliniques de la COVID-19 dans la population pédiatrique². La fièvre et la toux étaient des symptômes prépondérants dans plus de la moitié des cas, suivis par la rhinorrhée, la congestion nasale, la myalgie, la fatigue et le mal de gorge dans $10 \%-20 \%$ des cas, et par des symptômes gastro-intestinaux et le mal de tête dans moins de

\section{POINTS CLÉS}

- Les enfants infectés par le coronavirus du syndrome respiratoire aigu sévère 2 (SRAS-CoV-2) sont plus susceptibles d'être asymptomatiques ou de présenter des symptômes légers à modérés.

- Certains symptômes, comme la toux, la rhinorrhée et la fièvre, sont indiscernables de ceux d'autres maladies respiratoires virales.

- Il a été démontré que l'anosmie et l'agueusie sont fortement associées à l'obtention d'un résultat positif à un test de dépistage du SRAS-CoV-2, mais la valeur prédictive réelle de nombreux symptômes n'est toujours pas connue.

- Étant donné qu'aucune stratégie de dépistage de symptômes ne pourra empêcher tous les enfants infectés par le SRAS-CoV-2 d'entrer dans les écoles, il est essentiel de continuer d'insister sur d'autres mesures de santé et de sécurité - notamment la distanciation physique, l'hygiène des mains, le port du masque, l'amélioration de la ventilation et les activités d'apprentissage à l'extérieur - dans les écoles pour y prévenir la propagation de l'infection.

- D’autres études sont nécessaires pour comprendre la transmission secondaire du SRAS-CoV-2 chez les enfants et leurs familles, ainsi que les conditions de transmission.

$10 \%$ des $\mathrm{cas}^{2}$. Bien que l'anosmie, avec ou sans agueusie, se soit révélée être un symptôme propre à l'infection par le SRAS-CoV-2 chez près de la moitié des adultes atteints, King et ses collègues n'ont relevé l'un ou l'autre de ces symptômes que chez 7,7\% des enfants ayant été déclarés positiff ${ }^{1}$. Cette proportion s'apparente à ce qui a été observé dans une cohorte d'enfants de travailleurs de la santé au Royaume-Uni, mais est inférieure à celle de $20 \%$ associée à une cohorte pédiatrique en milieu hospitalier du Massachusetts ${ }^{3-5}$.

King et ses collègues proposent d'inclure seulement les symptômes associés à des résultats positifs dans les questionnaires de dépistage, mais reconnaissent que ceux-ci sont peu fréquents par rapport aux symptômes affectant les voies respiratoires supérieures et à la fièvre. Bien qu'ils aient calculé les rapports de vraisemblance positifs de la présence de chacun des symptômes dans leur analyse, leur approche ne fait que lier les symptômes à l'infection; elle ne 
montre pas si chacun s'est manifesté de façon isolée (p. ex. : la rhinorrhée seule, indépendamment de l'exposition, annonce-t-elle l'infection par le SRAS-CoV-2?) ni si un enfant présentant une rhinorrhée est susceptible d'obtenir un résultat positif à un test de dépistage du SRAS-CoV-2. Comme certains symptômes (p. ex. : mal de tête et rhinorrhée) sont courants chez les enfants d'âge scolaire même en l'absence d'infection, il serait important d'évaluer la valeur prédictive positive de chacun de ces symptômes pour la COVID-19, particulièrement en tant que symptômes isolés.

Dans ces conditions, quels symptômes devrait-on utiliser pour dépister les enfants atteints avant qu'ils n'entrent dans les écoles? L'utilisation d'une approche élargie au lieu d'une approche ciblée implique quelques compromis. Si l'on tentait de repérer tous les élèves qui pourraient avoir contracté le SRAS-CoV-2 avant qu'ils n'approchent de l'école en se fondant sur leurs symptômes, on risquerait d'exclure de nombreux élèves dont les symptômes ne se rapportent peut-être pas à la COVID-19, mais sont indiscernables de ceux de cette maladie. De plus, si on exigeait un test et l'isolement selon les résultats d'un dépistage portant sur un large éventail de symptômes, on pourrait dépasser rapidement la capacité de test et affaiblir la volonté des enfants et des parents de déclarer les symptômes. À l'inverse, si on se concentrait sur des symptômes spécifiques, mais peu courants, il se pourrait que des enfants ayant la COVID-19, mais n'ayant pas été diagnostiqués se présentent à l'école.

Puisqu'une grande proportion des enfants infectés par le SRASCoV-2 ne développent aucun symptôme, les stratégies de dépistage de symptômes ne peuvent vraisemblablement pas empêcher tous les enfants infectieux d'entrer dans les écoles. Par conséquent, les mesures de santé et de sécurité autres que le dépistage - notamment la distanciation physique, l'hygiène des mains, le port du masque, l'amélioration de la ventilation et les activités d'apprentissage à l'extérieur - qui y sont mises en place sont essentielles pour y prévenir la propagation de l'infection ${ }^{6-8}$. Il importe de recueillir des données sur la transmission secondaire à ces endroits pour évaluer ces mesures et les adapter selon les besoins.

L'utilisation d'un test de diagnostic rapide non effractif s'ajoutant aux mesures de santé publique rigoureuses visant le recours aux tests, la recherche des contacts et l'isolement des personnes infectées et de leurs contacts - pourrait faciliter le repérage des enfants infectés asymptomatiques ou présentant des symptômes légers, et favoriser le respect des recommandations à propos des tests et de l'isolement. De plus, la surveillance épidémiologique dans les réseaux, combinée à des analyses phylogénétiques des grappes de cas, pourrait s'avérer utile pour comprendre la transmission du SRAS-CoV-2 chez les enfants et leurs familles, ainsi que les conditions de transmission (p. ex. : durée, fréquence et intensité des expositions), particulièrement chez les porteurs asymptomatiques ${ }^{9}$. Même s'il a été démontré que les enfants ont une charge virale supérieure en début de maladie, il faudrait corréler ce résultat de laboratoire avec le risque de transmission tout au long de la maladie, en tenant compte de l'effet des mesures d'atténuation visant différents groupes d'âge (préscolaire, primaire et secondaire) ${ }^{4,10}$.

Le tableau clinique de l'infection par le SRAS-CoV-2 chez les enfants est souvent indiscernable de celui d'autres infections virales respiratoires, mais ses conséquences sur la présence à l'école sont considérables. Pour assurer l'allocation adéquate des ressources limitées aux mesures de santé publique et en milieu scolaire, il importe de comprendre et d'atténuer le plus possible les risques de transmission, en regard des avantages connus d'un apprentissage en personne ${ }^{11}$.

\section{Références}

1. King JA, Whitten TA, Bakal JA, et al. Symptoms associated with a positive result for a swab for SARS-CoV-2 infection among children in Alberta, Canada: an observational study. CMAJ 2020;193:E1-9.

2. Ontario Agency for Health Protection and Promotion (Public Health Ontario). Coronavirus disease 2019 and the pediatric population: an umbrella review. Toronto: Queen's Printer for Ontario; 2020. Accessible ici : www.publichealthontario .ca/-/media/documents/ncov/ipac/2020/10/covid-19-pediatric-population-umbrella -review-synthesis.pdf?la=en (consulté le 10 nov. 2020).

3. Ibekwe TS, Fasunla AJ, Orimadegun AE. Systematic review and meta-analysis of smell and taste disorders in COVID-19. OTO Open 2020;4: 2473974X20957975.

4. Yonker LM, Neilan AM, Bartsch Y, et al. Pediatric severe acute respiratory syndrome coronavirus 2 (SARS-CoV-2): clinical presentation, infectivity, and immune responses. J Pediatr 2020 Aug. 20 [Cyberpublication avant impression]. doi: 10.1016/j.jpeds.2020.08.037.

5. Waterfield T, Watson C, Moore R, et al. Seroprevalence of SARS-CoV-2 antibodies in children: a prospective multicentre cohort study. medRxiv 2020 Sept. 2 [préimpression]. doi: 10.1101/2020.08.31.20183095.

6. Heavey L, Casey G, Kelly C, et al. No evidence of secondary transmission of COVID19 from children attending school in Ireland, 2020. Euro Surveill 2020;25:2000903.

7. Macartney K, Quinn HE, Pillsbury AJ, et al. NSW COVID-19 Schools Study Team. Transmission of SARS-CoV-2 in Australian educational settings: a prospective cohort study. Lancet Child Adolesc Health 2020;4:807-16.

8. Tupper P, Colijn C. COVID-19's unfortunate events in schools: mitigating classroom clusters in the context of variable transmission. medRxiv 2020 Oct. 22 [préimpression]. doi: 10.1101/2020.10.20.20216267.

9. Lemieux J, Siddle KJ, Shaw BM, et al. Phylogenetic analysis of SARS-CoV-2 in the Boston area highlights the role of recurrent importation and superspreading events. medRxiv 2020 Aug. 25 [pré-impression]. doi: 10.1101/2020.08.23.20178236.

10. Heald-Sargent T, Muller WJ, Zheng X, et al. Age-related differences in nasopharyngeal severe acute respiratory syndrome coronavirus 2 (SARS-CoV-2) levels in patients with mild to moderate coronavirus disease 2019 (COVID-19). JAMA Pediatr 2020;174:902-3.

11. Christakis DA, Van Cleve W, Zimmerman FJ. Estimation of US children's educational attainment and years of life lost associated with primary school closures during the coronavirus disease 2019 pandemic. JAMA Netw Open 2020;2:e2028786.

Intérêts concurrents : Aucun déclaré.

Cet article a été sollicité et il n'a pas été révisé par des pairs.

Affiliations : Centre hospitalier pour enfants de l'est de l'Ontario (Thampi); Département de pédiatrie (Thampi), Université d'Ottawa, Ottawa, Ontario; Toronto Health Economics and Technology Assessment Collaborative (Sander), Réseau universitaire de santé; Institut des politiques, de la gestion et de l'évaluation de la santé (Sander), Université de Toronto; Hôpital SickKids (Science); Département de pédiatrie (Science), Université de Toronto, Toronto, Ontario.

Collaborateurs : Tous les auteurs ont contribué à la conception du travail, ont rédigé le manuscrit et en ont révisé de façon critique le contenu intellectuel important; ils ont donné leur approbation finale pour la version destinée à être publiée et assument l'entière responsabilité de tous les aspects du travail.

Propriété intellectuelle du contenu : Il s'agit d'un article en libre accès distribué conformément aux modalités de la licence Creative Commons Attribution (CC BY-NC-ND 4.0), qui permet l'utilisation, la diffusion et la reproduction dans tout médium à la condition que la publication originale soit adéquatement citée, que l'utilisation se fasse à des fins non commerciales (c.-à-d., recherche ou éducation) et qu'aucune modification ni adaptation n'y soit apportée. Voir : https:// creativecommons.org/licenses/by-nc-nd/4.0/

Correspondance : Nisha Thampi, nthampi@cheo.on.ca 